Supporting Information

\title{
Cellular Uptake and Cytotoxicity of Varying Aspect Ratios Gold Nanorods in HeLa Cells
}

\author{
Deshani Fernando, Shoukath Sulthana and Yolanda Vasquez *
}

* Email: yolanda. vasquez@okstate.edu

Department of Chemistry, 107 Physical Sciences I, Oklahoma State University, Stillwater, OK 74078, United States

\section{Determination of gold nanorod (GNRs) concentration}

Concentration of GNRs was determined by using a literature reported method with further modifications. ${ }^{1}$ Briefly, by using the images taken from TEM, the dimensions of each aspect ratio were measured and used to calculate the volume of a nanorod. For AR 11.5, volume was determined using equation (S1) assuming the cylindrical middle (h) having 2 spherical caps with height (a) and radius (r) as observed in TEM. Since no spherical caps were observed clearly for AR 2.6, AR 3.2 and AR 5.4, volume was determined only by considering the cylindrical middle with length (h). Assuming atom density of gold (D) (59 atoms $\left./ \mathrm{nm}^{3}\right)^{1}$ with the face-centered cubic crystal structure, the mass of Au per nanorod was calculated for each aspect ratio (equation S2) as shown in Table S1. By using the mass of Au per nanorod calculated previously and by analyzing the $\mathrm{Au}$ atom content of all four aspect ratios separately using inductively coupled plasma- atomic emission spectroscopy (ICP-AES), the GNR concentration was determined using equation (S3).

$$
\begin{array}{cl}
\text { Volume }(\mathrm{V})=\left(\frac{\pi}{3}\right) a\left(3 r^{2}+a^{2}\right)+\left(\pi r^{2} h\right) & \text { Equation (S1) } \\
\text { Mass of Au per nanorod }(\mathrm{g} / \text { nanorod })=\mathrm{V} \times \mathrm{D} \times 197 \mathrm{gmol}^{-1} /\left(6.022 \times 10^{23}\right) & \text { Equation (S2) }
\end{array}
$$


Gold nanorod concentration $($ nanorods $/ \mathrm{L})=\underline{\mathrm{Au} \text { atom concentration from } \mathrm{ICP}(\mathrm{g} / \mathrm{L})}$

Mass of Au per nanorod (g/nanorod)

Equation (S3)

After calculating the GNRs concentration, calibration curves were prepared by varying the concentration of GNRs and by observing the absorbance at $\lambda_{\max }$ for the three shorter rods (AR 2.6, AR 3.2 and AR 5.4) and at $520 \mathrm{~nm}$ for AR 11.5 by referring to a previous protocol. ${ }^{2}$ Before every experiment, the concentration of Au nanorods in each sample was determined using the calibration plots developed previously.

Table S1. Mass of Au per nanorod calculated using ICP-AES and TEM data.

\begin{tabular}{|l|l|}
\hline Aspect Ratio & $\begin{array}{c}\text { Mass of Au per } \\
\text { nanorod (g/nanorod) }\end{array}$ \\
\hline AR 2.6 & $7.00 \times 10^{-16}$ \\
\hline AR 3.2 & $2.36 \times 10^{-16}$ \\
\hline AR 5.4 & $1.10 \times 10^{-15}$ \\
\hline AR 11.5 & $2.58 \times 10^{-15}$ \\
\hline
\end{tabular}

\section{Stability of AR 3.2 and AR 11.5 GNRs in media}

To determine the effect of stability of GNRs in media on cellular uptake, CTAB functionalized GNRs of AR 3.2 and AR 11.5 were dispersed in complete media ( $80 \mathrm{pM}, 2 \mathrm{~mL})$ separately and the absorbance (at $770 \mathrm{~nm}$ for AR 3.2 and at $505 \mathrm{~nm}$ for AR 11.5) measured at different time intervals ( $3 \mathrm{~h}, 6 \mathrm{~h}, 10 \mathrm{~h}$ and $24 \mathrm{~h}$ ) using a UV-Vis spectrophotometer.

Table S2. Stability of CTAB functionalized GNRs of AR 3.2 and AR 11.5 in complete media.

\begin{tabular}{|c|r|r|r|r|}
\hline \multirow{2}{*}{$\begin{array}{c}\text { Aspect ratios of } \\
\text { GNRs }\end{array}$} & \multicolumn{4}{|c|}{$\begin{array}{c}\text { Change in the absorbance } \\
\text { (Initial absorbance - Final absorbance) }\end{array}$} \\
\cline { 2 - 5 } & $3 \mathrm{~h}$ & $6 \mathrm{~h}$ & $10 \mathrm{~h}$ & $24 \mathrm{~h}$ \\
\hline AR 3.2 in media & 0.009 & 0.014 & 0.019 & 0.037 \\
\hline AR11.5 in media & 0.023 & 0.023 & 0.033 & 0.084 \\
\hline
\end{tabular}


For CTAB stabilized samples of AR 3.2 and AR 11.5, a higher reduction in the absorbance was observed at a $24 \mathrm{~h}$ incubation time compared to the initial absorbance value. Reduction in absorbance values of AR 3.2 and AR 11.5 correlated well with the ICP data obtained at different time intervals as shown in Fig. 7A, where a significantly higher cellular uptake was observed at $24 \mathrm{~h}$ for AR 3.2 and AR 11.5 compared to shorter incubation times. Higher instability of AR 11.5 in media compared to the AR 3.2 indicates that a significantly higher cellular uptake of AR 11.5 is probably due to the sedimentation of the nanorods that promotes direct contact with the cells.

Size distribution of GNRs of AR 11.5 before and after sedimentation technique with $0.1 \mathrm{M}$ CTAB

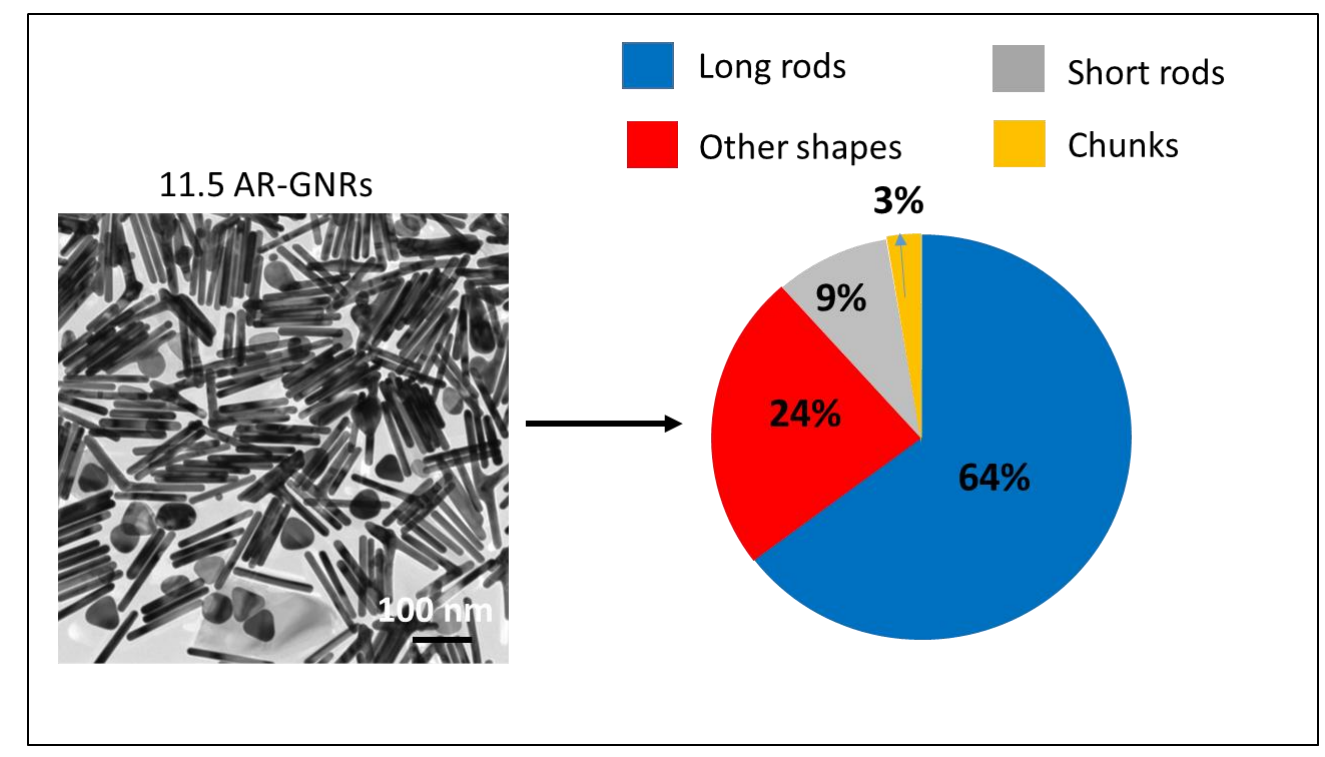

Figure S1. Distribution of GNRs from the synthetic protocol used to obtain particles of AR 11.5 as evaluated from TEM images. 


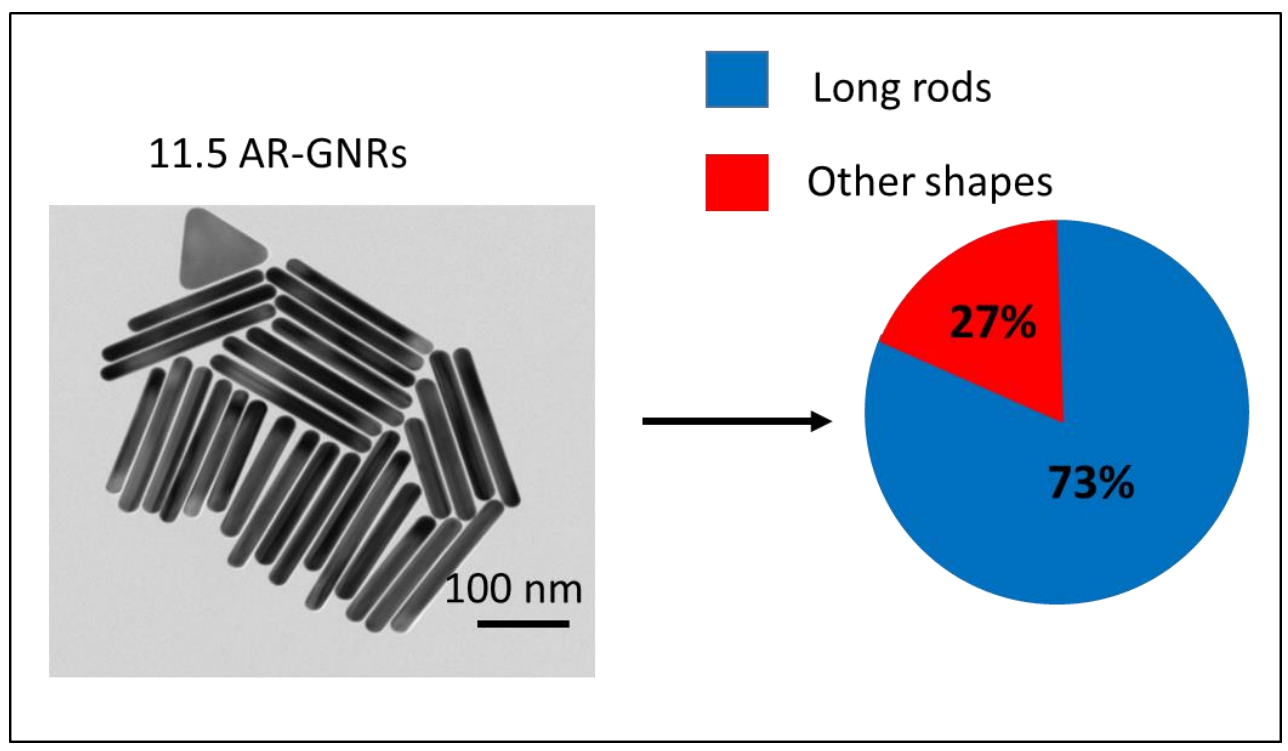

Figure S2. Distribution of GNRs from the synthetic protocol used to obtain AR 11.5 after sedimentation technique as purification method as evaluated from TEM images.
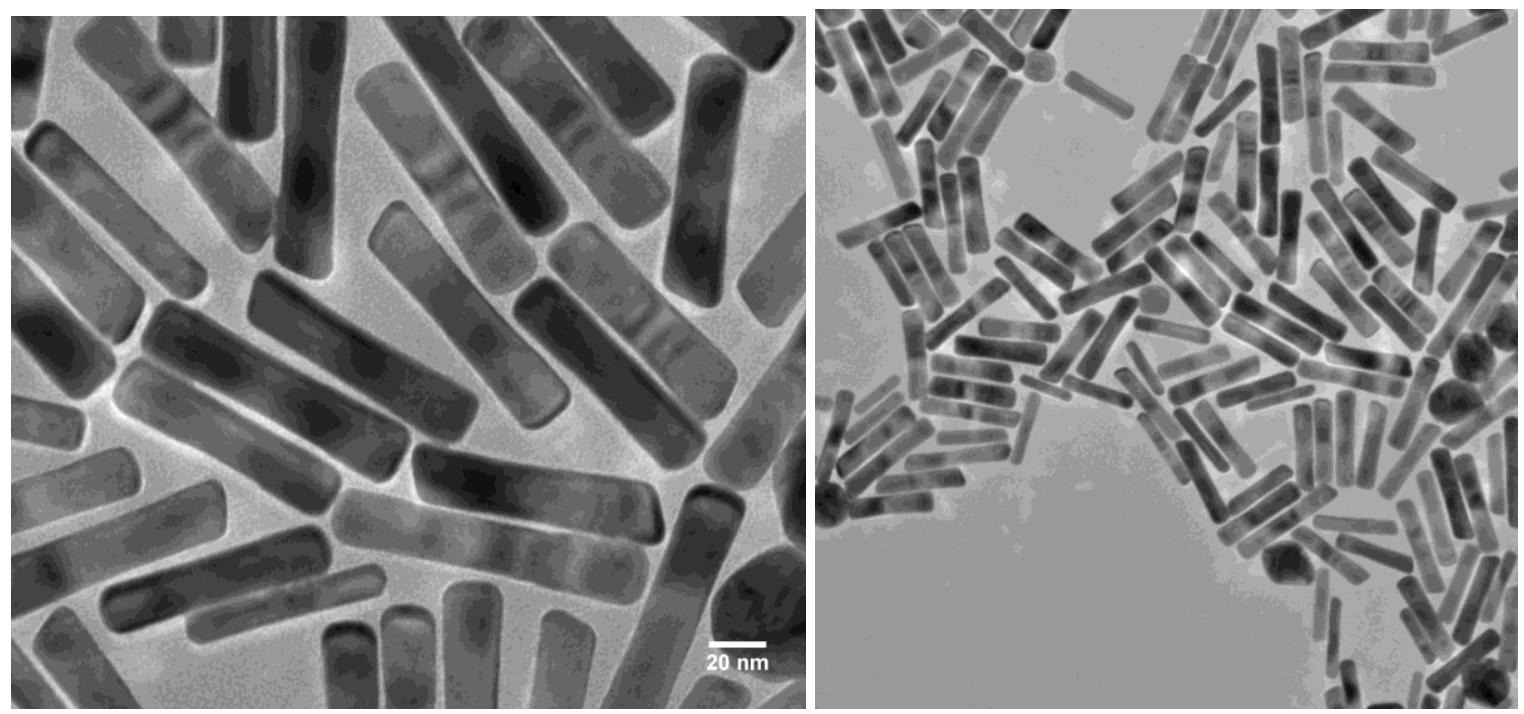

Figure S3. TEM images of GNRs of AR 5.4 after ligand exchange with PEG-SH.

\section{Determination of protein binding on to GNR surface}

Protein binding on to the Au nanorod surface having CTAB and PEG-SH were also studied by sodium dodecyl sulfate polyacrylamide gel electrophoresis (SDS-PAGE). After incubating GNRs (80 pM, $3.00 \mathrm{~mL}$ ) with different aspect ratios in complete media for $24 \mathrm{~h}$ having $10 \%$ DCS, each nanorod sample was centrifuged twice to remove unbound proteins present. Then, $\mathrm{NaCl}$ (1.00 
$\mathrm{M}, 50 \mu \mathrm{M}$ ) was added into each GNR sample and kept at room temperature overnight to separate the proteins bound to the surface of the GNRs. The supernatant of each GNR sample was separated by centrifugation ( $8000 \mathrm{rpm}, 30 \mathrm{~min}$.), and mixed with a sample buffer (containing Tris $\mathrm{HCl} \mathrm{pH}$ $6.8(1 \mathrm{M}), 30 \% \mathrm{w} / \mathrm{v}$ glycerol, $10 \% \mathrm{SDS}, 0.12 \%$ bromophenol blue and dithiothreitol $(0.93 \mathrm{mg})$ ), separately. This was heated $\left(100{ }^{\circ} \mathrm{C}, 10 \mathrm{~min}\right.$.) and the proteins adsorbed onto the surface were identified by electrophoresis with a gel containing $10 \%$ polyacrylamide.

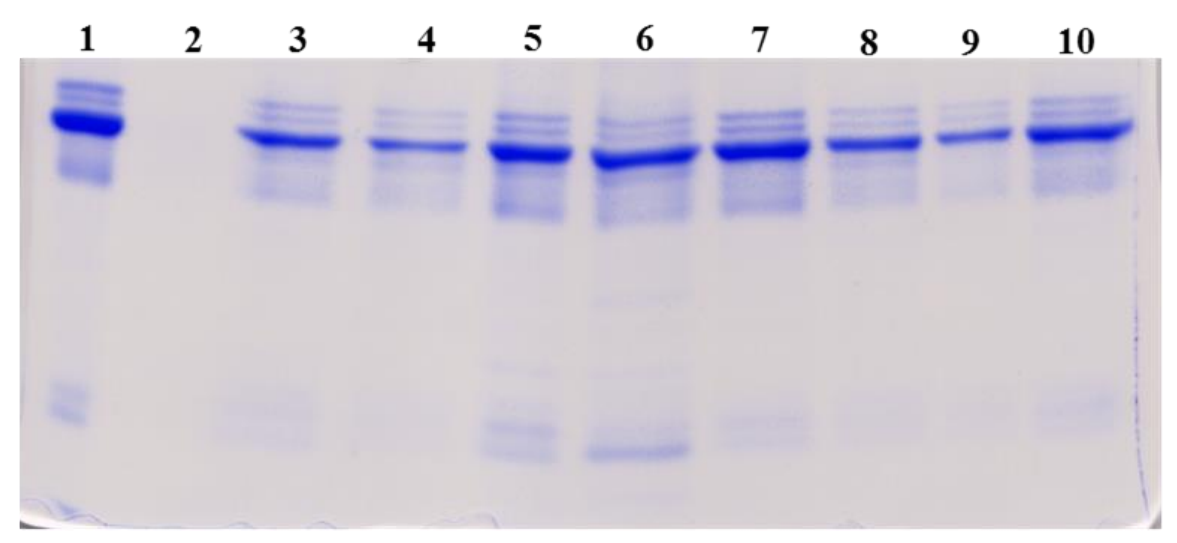

1. Media with DCS

2. Media without DCS

3. AR 2.6-CTAB

4. AR 3.2-CTAB

5. AR 5.4-CTAB

6. AR 11.5-CTAB

7. AR 2.6-mPEG

8. AR 3.2-mPEG

9. AR 5.4-mPEG

10. AR 11.5-mPEG

Figure S4. Bands observed in SDS-PAGE after incubating GNRs having CTAB and PEG-SH (80 $\mathrm{pM}$ ) in complete media with $10 \%$ DCS for $24 \mathrm{~h}$. The most intense band was recognized as serum albumin protein from the supernatant separated from different aspect ratios of GNRs. Complete media having 10\% DCS and DCS free media was used for the control experiments. 


\section{NIR absorption spectra of CTAB-stabilized GNRs for 11.5 AR}

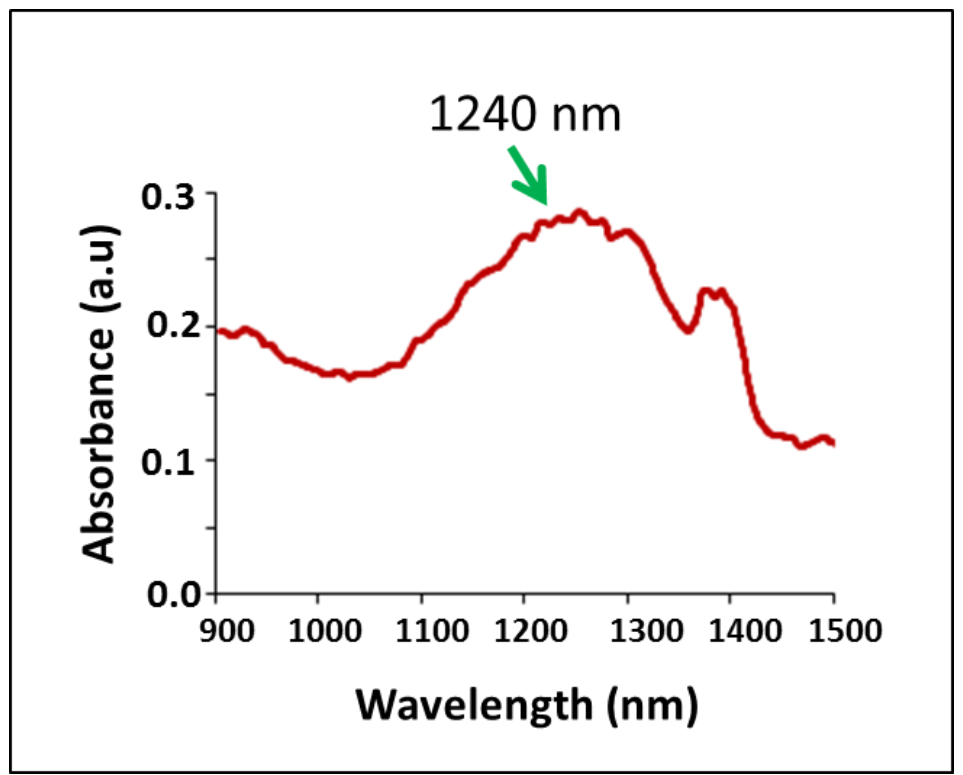

Figure S5. NIR absorption spectra of CTAB-stabilized GNRs for 11.5 AR.

\section{Determination of CTAB concentration in GNRs samples}

Concentration of free CTAB in GNRs samples was determined by using a literature reported method with minor modifications. ${ }^{3-5}$ Bromophenol blue (an anionic dye, BPB) was known to form a complex with cationic drugs, surfactants etc. ${ }^{3}$ The complex has a characteristic absorption at 606 $\mathrm{nm}$ as measured from UV-Vis spectrophotometry. A calibration curve was generated using different concentrations of CTAB solutions ranging from $3.5 \times 10^{-4} \mathrm{M}$ to $0.25 \times 10^{-4} \mathrm{M}$. The concentration of bromophenol blue was maintained at $1 \times 10^{-3} \mathrm{M}$. In a $20 \mathrm{~mL}$ glass vial, $5 \mathrm{~mL}$ of $\mathrm{CHCl}_{3}, 1 \mathrm{~mL}$ of CTAB working solution and $1 \mathrm{~mL}$ of $1 \times 10^{-3} \mathrm{M}$ BPB were added and stirred in a magnetic stirrer for $30 \mathrm{~min}$. Experiments were carried out in triplicates. After stirring for $30 \mathrm{~min}$, samples were placed undisturbed on the bench for 10 min to extract the BPB-CTAB complex (blue color) into $\mathrm{CHCl}_{3}$. Using a separatory funnel, the chloroform fraction was separated from the aqueous solution. Immediately, the samples were read at $606 \mathrm{~nm}$ against the corresponding blank solution using the UV-Vis spectrophotometry. 


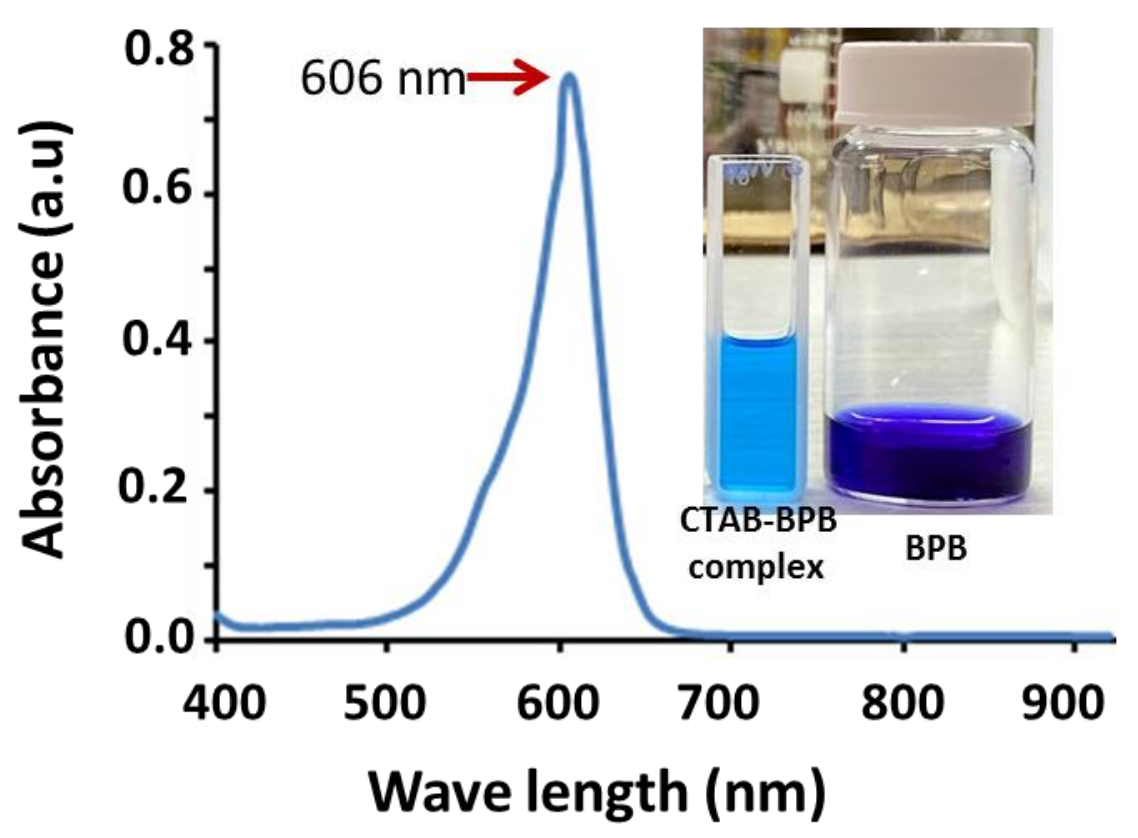

Figure S6. UV-Vis spectra of the complex formed from CTAB and BPB. The blue color complex has a characteristic absorbance at $606 \mathrm{~nm}$.

Table S3. Concentration GNRs of various aspect ratios was obtained from the supernatant after centrifugation of GNRs (free CTAB)

\begin{tabular}{|c|c|}
\hline GNRs $(80 \mathrm{pM})$ & Concentration $(\mathrm{mol} / \mathrm{L})$ \\
\hline $11.5 \mathrm{AR}$ & $1.64 \times 10^{-5}$ \\
\hline $5.4 \mathrm{AR}$ & $1.34 \times 10^{-4}$ \\
\hline $3.2 \mathrm{AR}$ & $5.45 \times 10^{-5}$ \\
\hline $2.6 \mathrm{AR}$ & $6.53 \times 10^{-5}$ \\
\hline
\end{tabular}




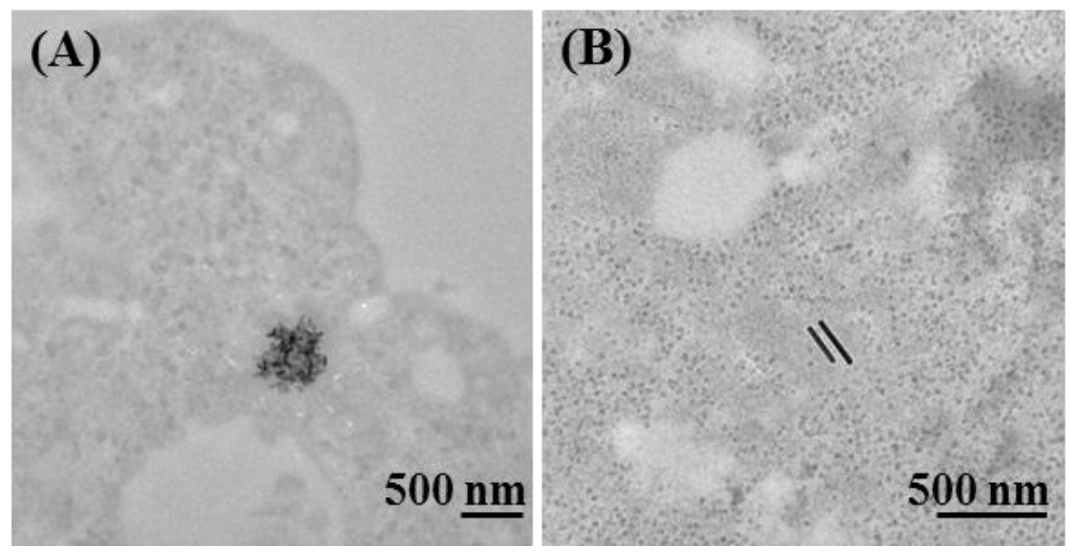

Figure S7. TEM images of PEG-SH functionalized Au NRs (80 pM) with ARs of (A) 5.4 and (B) AR 11.5 in the cytoplasm of HeLa cells.

References:

1.Vigderman, L.; Manna, P.; Zubarev, E. R. Quantitative Replacement of Cetyl trimethylammonium Bromide by Cationic Thiol Ligands on the Surface of Gold Nanorods and their Extremely Large Uptake by Cancer Cells. Angew. Chem. 2012, 124, 660-665.

2.Orendorff, C. J.; Murphy, C. J. Quantitation of Metal Content in the Silver-Assisted Growth of Gold Nanorods. J. Phys. Chem. B 2006, 110, 3990-3994.

3.Adura, C.; Guzman, F.; Alvarez-Lueje, A.; Kogan, M. J., Ion Pair Method to Determine the CTAB Content in Gold Nanorods Samples. J Chil Chem Soc 2014, 59 (4), 2701-2704.

4.Amjadi, M.; Manzoori, J. L.; Hassanzadeh, J., Surfactant to Dye Binding Degree Method for the Determination of Fluvoxamine Maleate and Citalopram Hydrobromide in Pharmaceuticals. Cent Eur J Chem 2010, 8 (3), 536-542.

5.Ramesh, P.; Basavaiah, K.; Rajendraprasad, N.; Devi, O. Z.; Vinay, K., Spectrophotometric Determination of Ofloxacin in Pharmaceuticals by Redox Reaction. J. Appl. Spectrosc. 2011, 78 (3), 383. 